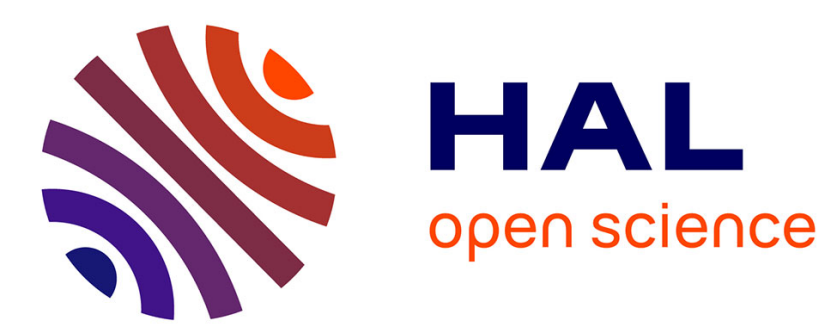

\title{
Universalismen zwischen Politik und Polizei. Jacques Rancière in der Postkolonie
}

\author{
Niklas Plätzer
}

\section{To cite this version:}

Niklas Plätzer. Universalismen zwischen Politik und Polizei. Jacques Rancière in der Postkolonie. Mareike Gebhardt. Staatskritik und Radikaldemokratie: Das Denken Jacques Rancières, Nomos Verlagsgesellschaft, pp.191-208, 2020, 978-3-8487-5918-7. 10.5771/9783748900474-191 . hal-03135358

\section{HAL Id: hal-03135358 https://hal.science/hal-03135358}

Submitted on 8 Feb 2021

HAL is a multi-disciplinary open access archive for the deposit and dissemination of scientific research documents, whether they are published or not. The documents may come from teaching and research institutions in France or abroad, or from public or private research centers.
L'archive ouverte pluridisciplinaire HAL, est destinée au dépôt et à la diffusion de documents scientifiques de niveau recherche, publiés ou non, émanant des établissements d'enseignement et de recherche français ou étrangers, des laboratoires publics ou privés. 


\section{Niklas Plätzer \\ Universalismen zwischen Politik und Polizei. Jacques Rancière in der Postkolonie}

Am 17. Oktober 1961 erklang der Ruf nach Algeriens Unabhängigkeit auf den Straßen von Paris. Zu Tausenden waren Demonstrant*innen gekommen, um ihre Solidarität mit dem Front de Libération Nationale (FLN) zu bekunden. „Dreißig, vierzigtausend Algerier*innen kamen plötzlich aus dem Boden geschossen, von den Grands Boulevards bis zum Quartier Latin, von Concorde bis zur Place de l'Étoi$l e^{\text {“1 }}$ (Péju et al. 2011, S. 21). Die Demonstrationen erschienen wie ein Befreiungsschlag nach Monaten der Repression in der Metropole und in Algerien - seit 1830 Kolonie und seit 1848 als drei départements unter französischer Herrschaft. 1961 waren im europäischen Teil Frankreichs 22 Polizist*innen durch FLN-Attentate ums Leben gekommen (Brunet 1999, S. 82), woraufhin Mitglieder polizeinaher Organisationen mindestens 173 algerische Aktivist*innen ermordeten (Brunet 1999, S. 137f.). Am 6. Oktober verhing der Polizeipräsident von Paris, Maurice Papon, eine Ausgangssperre, die sich explizit gegen ,muslimische Französinnen und Franzosen aus Algerien“ richtete (Brunet 1999 S. 164ff.). Als elf Tage später Zehntausende auf die Straße gingen, geschah dies als Akt des zivilen Ungehorsams gegen diese rassistische Maßnahme. Aber die Grausamkeit der Polizei übertraf alle Befürchtungen: Sie kam in Form von Schüssen, von ,dutzenden Toten, vielleicht zwischen 150 und 200“ (Seelow 2011), über 12.000 Festnahmen (Brunet 1999, S. 254) und unzähligen Folteropfern in der Hauptstadt des französischen Kolonialreichs.

Auch weite Teile der französischen Öffentlichkeit erlebten das Massaker vom 17. Oktober 1961 als einen Schock - darunter auch der 21-jährige Pariser Philosophiestudent Jacques Rancière, geboren als Sohn französischer Siedler in Algier. Unter dem Titel La cause de l'autre (Das Anliegen des Anderen) widmete er sich Jahrzehnte später den Ereignissen von 1961. Anlass für die bei Rancière seltene autobiographische Auseinandersetzung war die Konferenz France-Algérie. Regards croisés, die 1995 in Paris von Françoise Proust und Sidi Mohammed Barkat organisiert worden war (Rancière 1998, S. 256). ${ }^{2}$ Rancière (1998, S. 202) misst dem 17. Oktober 1961 nicht nur deshalb eine entscheidende Bedeutung bei, weil sich an diesem Tag

1 So berichtet die kommunistische und anti-koloniale Aktivistin Paulette Péju.

2 Soweit nicht anders angegeben sind alle Übersetzungen aus dem Französischen und Englischen meine eigenen. Hierbei habe ich, wo dies der Sinngehalt der Originalzitate zuließ, eine geschlechtergerechte Sprache gewählt. 
der „Sinn des Adjektivs ,französisch““ verändert habe und „das politische Subjekt ,Franzose“ von sich selbst abgespalten“" worden sei. Er lässt es vielmehr fast so erscheinen, als seien Grundbegriffe seiner politischen Theorie - Polizei, Politik, Desidentifikation - erstmals im Oktober 1961 im Kontext anti-kolonialer Proteste formuliert oder zumindest später von der eigenen Erinnerung an diese Zeit inspiriert worden.

Laut Rancière (1998, S. 211) sei der Algerienkrieg „kein Krieg“ gewesen, sondern eine „großangelegte Polizei-Operation.“ Doch verwendet er hier den Begriff der „Polizei“" schon wie im ebenfalls 1995 erschienenen La Mésentente im Sinne einer „Verteilung der Plätze und Funktionen“ und eines „System[s] der Legitimierung dieser Verteilung" (Rancière 2014a, S. 40). Wenn Rancière (1998, S. 211) den Algerienkrieg als eine „Polizei-Operation“ bezeichnet, dann geht es nicht bloß um repressive Gewalt, sondern um eine „Form des Eingriffs, die die Grenzen des Sichtbaren und des Unsichtbaren, des Sagbaren und des Unsagbaren bestimmt." Für Rancière bot der 17. Oktober 1961 nicht nur das grausame Schauspiel eines Polizeimassakers, sondern gab auch Einblick in eine koloniale Polizei, die auf die Unsichtbarkeit des anti-kolonialen Widerstands abzielte und letztlich auf das Ende von Politik überhaupt. „Politik,“ so Rancières (2014a, S. 41) berühmt-eigenwillige Definition, bezeichne jene Tätigkeit, die einen „Körper von dem Ort“ entferne, „,der ihm zugeordnet war oder die die Bestimmung eines Ortes ändert; sie lässt sehen, was keinen Ort hatte gesehen zu werden, lässt eine Rede hören, die nur als Lärm gehört wurde.“" Während in La Mésentente seine Beispiele noch die „Plebejer von Ballanche“ und „Arbeiter des 19. Jahrhunderts" betreffen, so entwickelt er in La cause de l'autre seinen ,anti-polizeilichen' Begriff von Politik im Kontext der algerischen Demonstrationen des 17. Oktober 1961.

Rancière (2014a, S. 9) zufolge kann Politik auf keine institutionelle Sphäre reduziert werden, sondern bezeichnet jene konflikthafte Praxis, „die als Prinzip die Gleichheit hat." Durch Politik bricht in der Ordnung ein Zwischenraum auf: der „Anteil der Anteilslosen“ distanziert den sozialen Raum von sich selbst, indem Hierarchien durch eine „Verifizierung“ der „Gleichheit zwischen Beliebigen“ aus den Angeln gehoben werden (Rancière 2014a, S. 50, 28). Rancières radikaldemokratische Perspektive ist wegen ihrer Parteinahme für marginalisierte Gruppen in einen Dialog mit zahlreichen politischen Kämpfen der Gegenwart gebracht worden: von der Migrant*innenpolitik (Beltrán 2009; Schaap 2011) zu Nuit Debout (Wahnich 2016), von der palästinensischen Intifada (May 2010) bis hin zu den gilets jaunes (Rancière, 2019), queeren Bewegungen (Chambers 2009; May 2009) und Black Lives Matter (Havercroft/Owen 2016). In all diesen Fällen ist von einem „Anteil der Anteilslosen" die Rede, der die Politik als performative Gleichheit gegen die Logik der Polizei ins Spiel bringe. Doch anstatt dieser Liste einen postkolonialen Eintrag hinzuzufügen, liegt es im Anschluss an La cause de l'autre nahe, die Formen und 
Grenzen des Rancièreschen Theorieapparats einer kritischen Analyse zu unterwerfen. ${ }^{3}$ Hierbei geht es um die Frage, inwieweit Rancières Politikverständnis als universalistisch zu verstehen ist und welche Probleme sich für einen Rancièreschen Universalismus ergeben, insofern dieser seinen Sinngehalt aus dem gesellschaftlichhistorischen Raum der französischen Postkolonie bezieht. ${ }^{4}$

Im Folgenden soll La cause de l'autre in einem ersten Schritt als Kritik sogenannter ,Identitätspolitik' gelesen werden. Rancière richtet sich hier mit deutlichen Worten gegen Sartre, Fanon und Bourdieu, die einen Krieg der Identitäten zum Paradigma der Politik erklärten. Solidarität ergebe sich aber gerade nicht aus der positiven Identifizierung mit einem Kampf um die Selbstidentität des Anderen, sondern, im Gegenteil, negativ durch die Desidentifizierung von der eigenen Identität. Anstatt Solidarität auf eine als prä-politisch vorausgesetzte (oder als telos von geschichtlicher Entwicklung immer schon anzustrebende) Identität zurückzuführen, ergibt sich politische Solidarität aus einer geteilten Erfahrung von Selbstverfremdung. Neben der historischen Verortung von Rancières Werk im Kontext der Dekolonisierung von Algerien leistet La cause de l'autre hierin einen Beitrag zur Begriffsklärung von Politik als Desidentifizierung.

Ein zweiter Teil untersucht, inwieweit Rancières Position als eine Neuformulierung einer französischen Universalismustradition verstanden werden kann und sich somit einer post- bzw. dekolonialen Kritik der Verstrickung von Universalismus in koloniale Herrschaft aussetzt. Die postkoloniale Kritik des Universalismus fragt danach, ob „das, was als ,universell“ bezeichnet wird,“ nicht „,das provinzielle Merkmal einer dominierenden Kultur" sei, deren „,Universalisierbarkeit" untrennbar [bleibe] von imperialer Expansion“ (Butler 2000, 15). Obgleich Rancière den republikanischen Universalismus in seinen fundamentalistischen Spielarten ablehnt, entwirft er selbst, so meine These, einen Universalismus der Zwischenräume. An die Stelle der transzendentalen Letztgründungsversuche des liberal-juridischen Universalismus einerseits und einer minoritär gewendeten Affirmation von Selbstidentität andererseits tritt die Inszenierung von Gleichheit durch die, die keinen Anteil haben: einer Gleichheit, die die Herrschaftsordnung wie einen Haarriss durchzieht, soziale Identitäten von sich selbst distanziert und im Spalt der Selbstdifferenzen die Möglichkeit einer „polemischen“ Universalität aufzeigen soll (Rancière 2014a, S. 50). In Nabila Abbas“ (2019, S. 395) Formulierung geht es Rancière um die „flüchtige, verschiebende, per definitionem nicht-identitäre Performanz der Politik." Doch wie ich in diesem Beitrag zeigen möchte, reduziert Rancière eine Vielfalt von Solidaritäts-

3 Die hier entwickelte postkoloniale Auseinandersetzung mit Rancière folgt Stoler (2011) und Toscano (2018).

4 „Gesellschaftlich-historisch“ ist im Sinne von Castoriadis” (1999) „social-historique“ zu verstehen. Mit „Postkolonie“ sind nicht nur ehemalige französische Kolonien gemeint, sondern die symbolische Institution von ,Frankreich“ als „,imperialer Formation“(Wilder 2015, S. 6f.). Zum Begriff der Postkolonie siehe auch: Mbembe 2001, S. 1-24. 
formen auf Desidentifizierung und privilegiert damit eine auf abstrakte Gleichheit gerichtete Emanzipationserzählung - ohne dabei zu beleuchten, ob diese Universalität nicht selbst in die Partikularität einer politischen Kultur zurückfällt. Es wäre zu fragen, ob nicht Identitätsbejahung im Kontext der französischen Postkolonie besser positioniert wäre, politische Effekte hervorzubringen. So kann durch die Affirmation dominierter subalterner Identitäten - im Sinne von Gayatri Chakravorty Spivaks (2006, S. 196-221, besonders S. 214) Konzept des „strategischen Essentialismus“ bei denen, die dominierende Identitätspositionen besetzen, ein Desidentifizierungsprozess ausgelöst werden. Dies wäre Identitätspolitik im nicht-pejorativen Sinn eines Rancièreschen Politikbegriffs.

Ein dritter Teil denkt schließlich mit Rancière gegen Rancière eine Form von anti-rassistischer Politik als Desidentifizierung von Weißsein. Hierfür ist es notwendig, in einer politischen Gemengelage, einer conjoncture politique, wie es Étienne Balibar (1992, S. 245; 1997, S. 65) immer wieder formuliert, bestimmte Des/Identifikationsdiskurse als politisch oder polizeilich bewerten zu können. Doch die Bestimmung einer politischen Strategie wird durch Rancières radikale Kritik an den Sozialwissenschaften sehr erschwert (Deranty/Genel 2016). So scheint es, dass sobald eine Differenzierung von Machtpositionen vorgenommen werden soll, eine empirische Analyse automatisch einem polizeilichen Einordnungsmanöver gleichkommen muss, dem politisches Handeln gerade widerstrebt. Die Soziologie von Rassifizierung, Klassenstrukturen oder Vergeschlechtlichung sei „Archipolitik“ und damit Komplize polizeilicher Herrschaft (Bosteels 2014, S. 80-92). Lois McNay (2014, S. 132-168) hat Rancières Ablehnung der Sozialwissenschaften scharf kritisiert: Sie führe zu einer social weightlessness, die für emanzipatorische Politik letztlich unbrauchbar werde. Welche Möglichkeiten und Grenzen ergeben sich aus Rancières abstrakter Gleichsetzung von Politik, Desidentifizierung und Universalismus vom Standpunkt dekolonialer und anti-rassistischer Solidarität?

\section{1. „Das Anliegen des Anderen”: Von der Kritik anti-kolonialer Revolution zur Politik als Desidentifikation}

La cause de l'autre beginnt mit einem Rückblick auf den philosophischen Anti-Kolonialismus im Frankreich der 60er Jahre.

\footnotetext{
„Wenn wir heute noch einmal die Schriften jener Autoren [sic] lesen, die 1960 das Anliegen der Algerier [sic] unterstützt haben, dann ist frappierend, zu beobachten wie der Philosoph Jean-Paul Sartre, in seinem Kommentar zu Frantz Fanon, und der Soziologe Pierre Bourdieu, ausgehend von seinen Erfahrungen der Feldforschung, in den Kategorien derselben Kosmologie argumentieren“ (Rancière 1998, S. 203f.).
} 
Laut Rancière handele es sich hierbei um eine kriegerische Weltanschauung: So ergreife Bourdieu Partei für einen anti-kolonialen Kampf um Selbstidentität, für den der Krieg, wie Rancière (1998, S. 204) zitiert, die „Masse der Landbevölkerung“ in einen „Wirbelsturm stürzt, der die Überbleibsel der Vergangenheit“ hinwegfege. Für Sartre sei, in paralleler Weise, der Krieg ,eine Zeit der Wahrheit, die durch das Stimme-Werden und das Volk-Werden eines von sich selbst entrissenen Subjekts den Abschluss einer historischen Form (des Kolonialismus) bedeutet" (Rancière 1998, S. 205). Damit werde der Krieg gegen den Kolonialismus zur Bedingung einer authentischen Identität der vormals Unterdrückten stilisiert.

In dieser Begeisterung für die anti-koloniale Revolution träfen sich zwei „entgegengesetzte Sartrismen“: das hegelianische „Denken einer Geschichtswahrheit“ und das existenzialistische Motiv einer „Freiheit, die aus dem Krieg des französischen Staates die Angelegenheit eines jeden Einzelnen“ machen wollte (Rancière 1998, S. 209). Sartre und Fanon, der von Rancière trotz eines direkten Bezugs auf Die Verdammten dieser Erde (1961) mit keiner theoretischen Auseinandersetzung gewürdigt wird, schwankten somit zwischen dem Paradigma des Krieges und dem der Moral. In beiden Fällen kritisiert Rancière einen fatalen Kurzschluss zwischen Politik und dem Kampf um die Erlangung einer authentischen Identität. Mit geschichtsphilosophischen und moralischen Argumenten sei die politische Dimension der Unabhängigkeitsbewegung aber gerade verpasst worden (Rancière 1998, S. 206). Rancière (1998, S. 215f.) geht sogar so weit, die Führer*innen der algerischen Bewegung als „Kompliz*innen“ in der „politischen Ausradierung [effacement] der Singularität“" ihres Kampfes zu bezeichnen. Die Idee der „Aneignung einer historischen Identität" sei mitverantwortlich dafür gewesen, dass zwischen Algerier*innen und Französinnen und Franzosen „keine starke politische Verbindung“ zustande gekommen sei: „Die Abstraktion des Anderen war die Antwort auf die Abstraktion des Eigenen“ (Rancière 1998, S. 215).

Indem er sich gegen einen Anti-Kolonialismus als Aneignung von Selbstidentität richtet, stellt Rancière (1998, S. 208) die Frage, ,inwiefern das Anliegen der Algerier [sic], unser Anliegen sein konnte und zwar anders als in moralischer Hinsicht." ${ }^{\text {5 }}$ An diesem Punkt richtet sein Vortrag den Blick auf die Ereignisse des 17. Oktober 1961. Dort seien drei Verhältnisse im Spiel gewesen: „zwischen den algerischen Aktivist*innen und dem französischen Staat, zwischen dem französischen Staat und uns und zwischen den algerischen Aktivist*innen und uns“ (Rancière 1998, S. 210). Was die Polizeioperation von Oktober 1961 deutlich mache, sei der negative Charakter ,politischer Subjektivierung“:

„Wir konnten uns als Demonstrant*innen im französischen öffentlichen Raum nicht mit den brutal in Erscheinung getretenen und dann brutal verschwundenen Algerier*innen

5 In einer eigentümlichen Fußnote erklärt Rancière (1998, S. 261): „,Wir‘ bezieht sich im Folgenden einfach auf eine politische Generation in ihrer Gesamtheit." 
identifizieren. Doch wir konnten uns des-identifizieren gegenüber einem Staat, der sie getötet und von jeder Rechnung abgeschrieben hatte" (Rancière 1998, S. 212).

An die Stelle eines anti-kolonialen Kampfes um Selbstidentität tritt bei Rancière solidarische Politik als Desidentifikation. Politische Subjektivierung ist somit nicht als ein $\mathrm{Zu}$-sich-selbst-Kommen zu verstehen, sondern als ein erfinderischer Prozess, in dem durch den Bruch mit einer sozialen Selbstidentität - in diesem Fall mit einem auf den Staat bezogenen ,Französischsein' - ein Überschuss produziert wird. Dieser Überschuss bezeichnet die Stelle, an der ein politisches Subjekt in Erscheinung tritt, das sich unterscheidet vom ,Volk ${ }^{6}$ der Polizei: von jenem ,Volk ${ }^{6}$, ,das vom Staat gesehen, benannt und gezählt“ wird (Rancière 1998, S. 212). Politische Subjektivierung bleibt demnach zugleich auf einen „Diskurs des Anderen” angewiesen: Sie „,beinhaltet eine unmögliche Identifikation, eine Identifikation mit einem Anderen, mit dem man sich gleichzeitig nicht identifizieren kann: ,Die Verdammten dieser Erde und andere" (Rancière 1998, S. 213). Im Oktober 1961 gab es laut Rancière (1998, S. 213) also keine Möglichkeit einer direkten Solidarität mit ,jenen Kämpfern, deren Gründe nicht die unseren waren und mit jenen Opfern, deren Gesichter für uns unsichtbar blieben." Stattdessen ergab sich die Erfahrung von Solidarität erst auf Umwegen, aus der Aktivierung eines „Bruchs zwischen Staatsbürgerschaft [citoyenneté juridique] einerseits und politischer Bürgerschaft [citoyenneté politique] andererseits." Es sei also durch die Desidentifizierung vom Staat, seiner Gewalt und der staatlich-polizeilichen ${ }^{6}$ Form der Bürger*innenschaft eine offene Form der Subjektivierung hervorgebracht worden, die eine politische Solidarität mit den algerischen Aktivist*innen ermöglicht habe.

Rancières Begriff der citoyenneté politique als Erfahrung einer offenen Solidarität durch Desidentifizierung unterscheidet sich damit in radikaler Weise vom republikanischen Modell der Staatsbürgerschaft, ,jenem ,Universalismus', der die Bürgerschaft vollständig mit dem staatlichen Rechtsrahmen identifiziert und selten die Gelegenheit verpasst, die Prinzipien der laïcité mit dem Schauder des Rassismus und die Verteidigung des Völkerrechts mit Eroberungsfieber zu verbinden“" (Rancière 1998, S. 218). Alberto Toscano (2018) unterstreicht, dass es die Auseinandersetzung mit den ,antinomies of citizenship“ gewesen sei, die der Generation von Rancière und Balibar den Rahmen bot, postkoloniale Fragen in ihr Denken einzubeziehen. Die Hinwendung zur citoyenneté als politische Subjektivierung ist bei Rancière und Balibar besonders auf die Konferenz France-Algérie. Regards croisés von 1995 zurückzuführen, an der beide teilnahmen (Balibar 1997, S. 5-22). Während für Sartres Generation noch die Frage revolutionärer Gewalt im Vordergrund stand, so entwickeln Balibar und Rancière eine politische citoyenneté in der Auseinandersetzung

6 In La cause de l'autre verwendet Rancière die Begriffe État und police weitestgehend synonym. Er hat aber später klargemacht, dass der Staat nicht zwangsläufig auf Polizei zu reduzieren sei, sondern auch zum Schauplatz von Politik werden kann (Rancière 2016, S. 166f.). 
mit dem Massaker vom 17. Oktober 1961 (Toscano 2018). ${ }^{7}$ Hierbei geht es ihnen nicht um „Chroniken der Entzauberung" [chroniques du désenchantement] als Antwort auf einen enttäuschten „historischen Glauben“ an die Revolution: Die citoyenneté ist keine Ersatzerzählung für Ex-Revoluzzer (Rancière 1998, S. 216f.). Vielmehr ist es Rancières Ziel, mit philosophischen Letztbegründungsversuchen zu brechen, um der Singularität und Kontingenz des politischen Handelns überhaupt erst gerecht werden zu können. Die Singularität der Politik lag im Oktober 1961 in der Desidentifikation junger Französinnen und Franzosen vom kolonialen Staat, die einen „Unterschied der citoyenneté zu sich selbst“ demonstrierte (Rancière 1998, S. 219). Die Ereignisse, die als „Algerienkrieg“ bezeichnet werden, erscheinen Rancière (1998, S. 219) hingegen als die Produktion des „Vergessens von innerer Alterität" und gerade darum als eine Polizeioperation, die bis heute anhält.

\section{Desidentifikation als universelle Gleichheit: Formen und Grenzen der Solidarität bei Rancière}

Politische Subjektivierung als Desidentifikation ist für Rancière ein Gleichheitsvorgang. Sie bringt eine nicht hierarchisch oder identitär vorgefasste citoyenneté auf die Bühne, ,verifiziert' die Annahme universeller Gleichheit und inszeniert das Ereignis von Demokratie - drei Begriffe, die er bis zur Ununterscheidbarkeit miteinander in Verbindung bringt. Während es eindeutig erscheint, welche Rolle der universalistische Gleichheitsbegriff für Politik als Desidentifikation spielen soll - nämlich die der Denaturalisierung von Hierarchien -, so sind die Kommentator*innen von Rancières Werk unterschiedlicher Auffassung, wenn es darum geht, ihren „,normativen Status" in einem konzeptuellen Gefüge auszumachen, das ohne letztbegründete Argumente auskommen soll (Deranty/Genel 2016, S. 57). An diesem Punkt kann zwischen drei Lesarten unterschieden werden: In einer ersten Lesart gilt Gleichheit bei Rancière als universelle Komponente von Sprache oder menschlicher Intelligenz, da noch im Befehl des Herrschenden eine Gleichheit vorausgesetzt sei, die das Verständnis des Befehls als Befehl ermögliche (Rancière 2014a, S. 55-72; Russell/ Montin 2015). Diese „Gleichheit der Intelligenz“ wird durch politisches Handeln aktualisiert und destabilisiert als Hintergrundannahme jede Form von Hierarchie (Fjeld

7 Der (von Rancière erwähnte) historische Hintergrund für Sartres Auseinandersetzung mit kolonialer und anti-kolonialer Gewalt ist einerseits das Massaker von Sétif, bei dem am 8. Mai 1945 zwischen 15.000 und 20.000 algerische Demonstrant*innen von französischer Polizei und kolonialen Milizen ermordet wurden, andererseits die Schlacht von Algier (1956-1957), wo der Front de Libération Nationale einen Guerillakrieg gegen die französische Kolonialmacht führte, mit etwa 300 französischen und zwischen 1.000 und 3.000 algerischen Todesopfern. Zu Sétif, s. Peyroulou 2008. Zur Schlacht von Algier, s. Harbi/Stora 2004, S. 489. 
2018, S. 39-75). ${ }^{8}$ Dies kann als fundamentalistische Lesart bezeichnet werden. So schreibt Abbas (2019, S. 395), dass Rancière „aus der radikalegalitären Intelligenz der Menschen [...] ihr radikalegalitäres Anrecht auf politische Teilhabe" ableite. Eine ähnliche, fast aristotelische Interpretation findet sich bei May:

„Frauen, Schwule und Lesben, Afro-Amerikaner*innen und andere können alle im Namen der Gleichheit handeln, weil sie alle in der Lage sind, gemeinsam ein sinnerfülltes Leben zu führen - d.h. alle sind sprechende Wesen - und alle sollten mit dem Respekt behandelt werden und andere mit dem Respekt behandeln, der in dieser Fähigkeit begründet liegt“" (May 2009, S. 11).

Diese fundamentalistische Lesart scheint Rancières Gleichheitsannahme aber als genau das zu positionieren, was er andernorts als archi- bzw. para-politische Festschreibung einer gerechten Ordnung von sich weist und als moralphilosophische Spielarten der Polizei ablehnt (Chambers 2013, S. 59-66). So argumentiert Myers (2016, S. 47f.), dass bei Rancière Gleichheit in „Beweis-Ereignissen” (demonstrative events) in Erscheinung trete, durch die eine ,universelle, aber verleugnete Bedingung" enthüllt würde. Doch eine solche Vorstellung von Gleichheit als der verborgene Grund sozialer Hierarchien ist mit einem Rancièreschen Politikbegriff nicht zu vereinbaren: Egalitäre Politik als radikal-erfinderische Intervention kann nicht auf die Verwirklichung einer ohnehin ,natürlich' bestehenden Gleichheit zurückgeführt werden. Wie Chambers (2013, S. 26f.) bündig formuliert: „Für Rancière ist Gleichheit kein Ordnungsprinzip, sondern ein Mechanismus der Unordnung.“

Die post-fundamentalistische Lesart gründet, zweitens, den universalistischen Gehalt der Rancièreschen Gleichheitsannahme nicht im Wesen der Sprache oder in gleicher Intelligenz, sondern sieht bei Rancière einen ,emanzipatorischen Apriorismus" am Werk, der ,letztlich nur axiomatisch [...] behauptet werden kann“ (Marchart 2010, S. 183). Doch während ein non sequitur der Rancièreschen Gleichheitsbehauptung von Marchart (2010, S. 183f.) beklagt wird, verteidigt Zerilli (2005, S. 63) gerade seine Unableitbarkeit: „Die Theorie, die der Politik angemessen ist, [...] ist selbst ein Handeln und kein Wissen, das die Politik von außen anleiten könnte." Dies muss auch für Rancières Annahme universeller Gleichheit gelten; genau deshalb spricht Rancière (2009) auch stets von einer „Behauptung“ (présupposition) von Gleichheit statt von einer Tatsache (Chambers 2013, S. 25-31). Die Frage nach den „normativen Ressourcen“, die den Dialog zwischen Rancière und Axel Honneth ${ }^{9}$ bestimmt hat, kann nicht als eine der politischen Handlung vorausgehende Frage formuliert werden, ohne selbst die Politik als Politik zu gefährden (Deranty/ Genel 2016, S. 16). Flügel-Martinsen (2015) bringt dieses Problem auf den Punkt, wenn er von einer „Normativitätsbegründungsfalle“ spricht. Damit ist Rancières

8 Fjeld (2018, S. 6ff.) unterscheidet bei Rancière zwischen drei Gleichheitsbegriffen (intellektuell, politisch und ästhetisch), die auf verschiedene Weise behauptet bzw. begründet würden.

9 Siehe dazu auch den Beitrag von Matthias Flatscher und Sergej Seitz in diesem Band. 
Gleichheitsbegriff gerade kein „fundamentalistischer Restbestand“, wie Marchart (2010, S. 184) meint, sondern Resultat einer konsequenten Weigerung, die politische Dimension der polemisch behaupteten Gleichheit wieder philosophisch einzufangen und damit zu untergraben - etwa durch eine Ontologie des Antagonismus. ${ }^{10}$

So überzeugend die post-fundamentalistische Lesart eine philosophische Begründung von Gleichheit vermeidet, so kann sie Rancières Gleichheitsbegriff nicht von seinen gesellschaftlich-historischen Umständen lösen. Diese stehen im Mittelpunkt einer provinzialisierenden Lesart. Dipesh Chakrabarty (2009, S. xiii) folgend bedeutet „Provinzialisieren“

„herauszufinden, wie und in welchem Sinn europäische Ideen, die als ,universell` galten, zugleich aus einer sehr partikularen intellektuellen und historischen Tradition bezogen worden sind, die keine universelle Gültigkeit behaupten kann. Dies bedeutet, die Frage zu stellen, was das Denken mit seinem Ort zu tun hat. Kann Denken seinen Herkunftsort transzendieren? Oder lässt der Ort einen Abdruck im Denken, der auf diese Weise die Idee einer vollständig abstrakten Kategorie in Frage stellt?“

Die Provinzialisierung von Rancières politischer Theorie heißt nicht etwa, die Radikalität seines Entwurfs aufgrund historischer Determiniertheit zu verwerfen, sondern zu untersuchen, inwieweit der „emanzipatorische Apriorismus“ (Marchart 2010, S. 178) Politik in einem Kontext transzendierenden Sinn definiert, der selbst wiederum Ausdruck einer partikularen Kultur bleibt. Damit würde sich der prinzipiell ,leere Universalismus Rancièrescher Politik als Echo einer französischen Tradition erweisen - und damit wiederum selbst als Ausdruck einer ,polizeilichen' Identität. Wie bei Lefort (1986, S. 30), der die „demokratische Erfahrung“ als Errungenschaft einer europäischen Entwicklung von Säkularisierung und der „Desinkorporation“ des Sozialen ansah, so scheint auch Rancières Gleichheitsbegriff - obgleich weniger explizit - auf einen normativen Eurozentrismus zu verweisen, insofern eine spezifische Emanzipationserzählung zum Merkmal von Politik überhaupt universalisiert wird. ${ }^{11}$ Balibar folgt dieser provinzialisierenden Lesart bereits 1995 in seinem Beitrag zur Konferenz France-Algérie. Regards croisés unter dem Titel Algérie, France - une ou deux nations? (Algerien, Frankreich - eine oder zwei Nationen?):

„Jacques Rancière sagt uns: vom Verhältnis zwischen Frankreich und Algerien zu sprechen, bedeutet zuallererst, vom Verhältnis Frankreichs, zu sich selbst' zu sprechen, zur Alterität, die es beinhaltet und die es zu einem großen Teil verleugnet. Dies heißt, die Frage nach der Desidentifizierung zu stellen, ohne die es keine demokratische Politik geben kann. Für ihn wie für mich ist es aufgrund unserer Nationalität schwierig, auf die andere Seite zu wechseln [passer sur l'autre bord]. Aber müssen wir das nicht tun, hier und

10 In der Absage an eine Ontologie des Politischen unterscheidet sich Rancières Spielart des Postfundamentalismus deutlich von Chantal Mouffes (2005, S. 8-35) Theorie radikaler Demokratie, die aus der These eines ontologischen Antagonismus Schlüsse für das politische Handeln zieht.

$11 \mathrm{Zu}$ Leforts Idee der westlichen Moderne als historische und zugleich vorgeblich „quasi-transzendentale" Bedingung von Demokratie siehe Marchart 2000, S. 78ff. 
jetzt? [...] Dennoch, jene Diskurse der Desidentifizierung, durch die sich ein jeder versucht, von seiner ihn gefangen haltenden Identität zu befreien und die Alterität anzuerkennen, die ihn ausmacht - könnten diese Diskurse ein einziger Diskurs sein, oder wenigstens ein gemeinsamer Diskurs, ein geteilter Diskurs? Es ist nicht auf abstrakte Weise, sondern auf Basis einer konkreten, schmerzhaften Erfahrung, dass wir eine Form der Gegenseitigkeit finden müssen“ (Balibar 1997, S. 8).

Obwohl Balibar genau wie Rancière die Solidarität mit den algerischen Demonstrant*innen als einen mit citoyenneté benannten Desidentifikationsprozess denken will, so unterstreicht er zugleich, dass eine Vielzahl von Diskursen der Desidentifizierung keinesfalls durch Abstraktion auf universelle Gleichheit reduziert werden können. Im Gegensatz zu Rancière, dessen Auseinandersetzung mit postkolonialen Fragen nach 1995 wieder abbricht, steht das komplexe Verhältnis von pluralen historischen Erfahrungen und universalistischer Politik bis heute im Zentrum von Balibars (2016) Denken.

Für Rancière jedoch bleibt der Weg einer historischen Diskursanalyse weitestgehend verschlossen, da die sozialwissenschaftliche Betrachtung von Machtverhältnissen das Risiko liefe, in eine die Politik eliminierende Polizeilogik umzuschlagen. Dabei ist es Rancières Unwille, seine eigene Position als weißer französischer Philosoph in der postkolonialen Metropole in den Blick zu bekommen, die seinen abstrakten Gleichheitsbegriffs in die Nähe eines, differenzblinden' Universalismus à la française bringt. Insofern Rancières Gleichheitsbehauptung gerade nicht philosophisch als Ordnungsprinzip, sondern politisch als „Mechanismus der Unordnung“ (Chambers 2013, S. 26f.) verstanden werden soll, muss sie sich auch einer politischkontextuellen Kritik aussetzen. Genau auf dieser Ebene verfehlt die polemische Intervention des Rancièreschen Universalismus aber ihr Ziel, indem sie die Verstrickung französischer Universalismus-Diskurse in die Reproduktion (post)kolonialer Hierarchien systematisch ausblendet.

\section{3. „Wie Sie vielleicht wissen, bin ich Franzose“: Gleichheit unter postkolonialen Bedingungen}

Rancières Unverständnis gegenüber der Bedeutung des strategischen Essentialismus für anti-koloniale und anti-rassistische Bewegungen ist in seinem Begriff der Politik als Gleichheitsvorgang angelegt. So richtet La haine de la démocratie (2005) zwar den Blick auf Rosa Parks, die sich 1955 weigerte, in Montgomery, Alabama ihren für weiße Busfahrgäste reservierten Sitzplatz aufzugeben (Rancière 2006, S. 136f.). Doch wird dieser Akt in Rancières Perspektive als eine Affirmation universeller Gleichheit gewertet und von jedem substantiellen Bezug zu Rassifizierung losgelöst - etwa auch die Tatsache, dass die Aktivistin Parks sich selbst später der Black 
Power-Bewegung anschloss. ${ }^{12}$ Für Rancière (2006, S. 136) geht es in Rosa Parks' Widerstand, gemäß seines abstrakten Politikbegriffs, um die differenzblinde Gleichheitsbehauptung einer „Bürgerin der Vereinigten Staaten, die sich entschloss, die Rechte zu haben, die sie nicht hatte.“

Die Frage, welche spezifische Hierarchie durch eine citoyenneté politique jeweils unterbrochen wird, stellt sich für Rancière nicht. Im Gegensatz zu Balibars Sensibilität für historisch situierte Diskurse der Desidentifizierung verschmelzen Rancières Fallbeispiele vor dem Horizont einer abstrakten Universalität. So erscheinen Französinnen und Franzosen, die sich vom Staat desidentifizieren, als äquivalent zu algerischen Aktivist*innen, und Rosa Parks wiederum als austauschbar mit Olympe de Gouges (Rancière 2006, S. 135). Konkrete Unterdrückungserfahrungen dürfen gerade nicht in Erscheinung treten, denn sie liefen Gefahr, vom Zwischenraum egalitärer Politik in die Echokammer prä-politischer Identitäten abzugleiten. Vor diesem Hintergrund kann es nicht verwundern, dass Rancière 2005 die massiven Banlieue-Unruhen als identitär und unpolitisch kritisiert hat und sich einer Solidarisierung mit den mehrheitlich nicht-weißen Demonstrierenden verweigerte (Gündoğdu 2017, S. 208). Rancières frappierende und zugleich für die französische Debatte kaum ungewöhnliche Weigerung, die Affirmation einer subalternen Identität als Politik ernst $\mathrm{zu}$ nehmen, erscheint hierbei als eine Form von rassifizierter Blindheit, wie sie in epistemologischen Debatten der critical race theory seit langem diskutiert wird.

Bereits in Schwarze Haut, weiße Masken (1952) liefert Fanon (2008, S. 152f.) nicht nur eine phänomenologische Analyse der Entmenschlichung von als ,schwarz rassifizierten Subjekten durch ,Weiße‘, die in einem schwarzen Gesicht nur ,exotische" oder animalische Züge wahrnehmen können; wie José Medina hervorgehoben hat, richtet sich Fanon auch gegen eine weitere Form des „weißen Unwissens“ (white ignorance), nämlich gegen jene wohlgemeinte Blindheit, die selbst Anti-Rassist*innen wie Sartre (oder Rancière) befallen könne: das Vergessen der Menschlichkeit des Schwarzen ,in seiner Differenz, [...] in der Spezifik der schwarzen Erfahrung des Leids“ (Medina 2013, S. 151). „Jean-Paul Sartre hat vergessen, dass der Schwarze in seinem Körper sehr anders leidet als der weiße Mann“, schreibt Fanon (2008, S. 117). Medina (2013, S. 150-154) zufolge sorgt die color blindness eines abstrakten Universalismus dafür, jene Bedingungen auszublenden, die überhaupt erst das Privileg begründen, von spezifischen Unterdrückungserfahrungen abstrahieren zu können. Dies sei die Meta-Blindheit (meta-blindness) differenzblinder Politik: ein Abstumpfen gegenüber den Bedingungen des Abstrahierenkönnens, aber auch ein Ausblenden hierarchischer Machtstrukturen, die durch universalistische Diskurse gestützt werden (Medina 2013, S. 75f., 150ff., 209-212). Solche Meta-Blindheit gewinnt an zusätzlicher Bedeutung im Nachwirken französischer Kolonialdiskurse, die

12 „Parks's beliefs and activities [...] challenge the sharp line often drawn between the civil rights and Black Power struggles“, schreibt z.B. Theoharis (2013, S. 203). 
eine mission civilisatrice gegenüber, wilden 'Völkern im Globalen Süden nicht etwa als Widerspruch zur revolutionären Tradition Frankreichs, sondern explizit als ihre Fortführung angesehen haben (Conklin 1997, S. 1-38).

Auf der Ebene begrifflicher Abstraktion ist sich Rancière (2009, S. 118) auch durchaus bewusst, dass „Politik fast überall und zu jeder Zeit mit der Polizei verbunden oder sogar mit ihr verwechselbar ist." Gegen anti-institutionelle Lesarten hat Chambers (2013, S. 38-65, 173) deutlich gemacht, dass Rancières Politik „unrein“ und mit den Institutionen und Machtstrukturen, gegen die sie sich richtet, „,verknotet" bleibt. ${ }^{13}$ Doch scheint Rancière nicht auf den Gedanken zu kommen, dass seine Äquivalenz von Desidentifizierung und Universalismus - sein eigener Politikbegriff - selbst einer Polizeilogik zum Verwechseln ähnlich werden könnte. So ist es seiner Meta-Blindheit geschuldet, dass er 1999 in einem Interview für die Quebecer Zeitschrift Possibles die sozial-historische Determiniertheit seines Gleichheitsbegriffs anerkannt und deren Bedeutung im selben Atemzug verworfen hat:

„Politik beginnt, wenn man nicht mehr der Repräsentant einer partikularen lokalen, religiösen oder sozialen Gemeinschaft ist. Politik beginnt, wenn man endlich eine Identifizierung mit Anonymität erreicht. [...] In Frankreich kämpfen Gruppen nicht um ihre Rechte als Gruppen, sondern um eine Art universelles Recht. Schauen Sie sich den Unterschied zwischen den feministischen Bewegungen in Frankreich und Nordamerika an. [...] Frankreich ist ein Land, in dem alle spezifischen Kämpfe die Form eines Kampfes um Gleichheit nehmen können" (Rancière 2014b, S. 140f.).

Rancières Selbstwiderspruch liegt darin, dass Politik in der Desidentifizierung von prä-politischen Identitäten liegen soll, aber genau dieser Politikbegriff von ihm zum Ausdruck einer spezifisch französischen Tradition erklärt wird. Im Gegensatz zu Balibar, für den auf den ersten Schritt der Selbstprovinzialisierung eine reflexive Analyse von historisch situierten Universalismen im Plural folgen muss, beharrt Rancière (2014b, S. 140) darauf, dass Politik zugleich eine „Identifizierung mit Anonymität" bedeute - nicht etwa mit einem Anders-Selbst-Sein (Anders-Frau-Sein, AndersSchwarz-Sein, etc.). Obwohl Rancière (2014b, S. 141) sich darüber im Klaren ist, dass „ein winziges Segment der Bevölkerung“ über „eine Art Monopol über die Universalität" verfügt, bezieht er diesen Befund an keiner Stelle auf sich selbst. Eine Meta-Blindheit für die Bedingungen und Effekte seines universalistischen Gleichheitsbegriffs verschließt ihm darum auch den Blick auf die Pluralität feministischer Diskurse in Frankreich, die, wie Françoise Vergès, Hourya Bentouhami und Elsa Dorlin gezeigt haben, keineswegs auf die stereotype Vorstellung eines universalistischen Feminismus reduziert werden können. ${ }^{14}$ Sudeep Dasguptas Frage in einem Interview von 2008, wie sich die Kritik der Selbstidentität zum Feld der postkolonialen Studien verhalte, hat Rancière noch einmal mit dem widersprüchlichen Versuch

13 Rancières Politik ist also keineswegs mit dem Bruchmoment einer Revolution zu verwechseln. 14 Siehe exemplarisch Vergès 2019; Bentouhami 2018; Dorlin 2017. 
beantwortet, seine politische Position (seine Theorie als politische Intervention) in seiner polizeilichen Identität (seinem Französisch-Sein) begründen zu wollen:

„Wie Sie vielleicht wissen, bin ich Franzose [lacht]. In Frankreich gibt es keine Identitätspolitik, da gibt es keine postkolonialen Studien. Die werden in Frankreich systematisch ignoriert. Von daher ist meine Auseinandersetzung mit der Frage des Subjekts auch nie der Versuch gewesen, Fragen der Identitätspolitik oder hybrider, postkolonialer Identitäten usw. zu berücksichtigen“" (Dasgupta, 2008, S. 76).

Diese Aussage ist nicht nur unzutreffend, sondern geradezu Ausdruck einer Sprechblockade - einer „kolonialen Aphasie“, wie Ann Laura Stoler (2011, S. 131) es in Bezug auf Rancière formuliert hat. Medina folgend kann Rancières eigene Unfähigkeit, sich selbst im Raum der französischen Postkolonie zu verorten, als Effekt seines differenzblinden Universalismus interpretiert werden. Die Erfahrungen anti-rassistischer Bewegungen zeigen in besonderer Weise, dass jene Subjekte, denen die Polizeiordnung die Position des ,Weißen' zugewiesen hat, durch universalistische Diskurse kaum für Solidarität zu sensibilisieren sind. Im Gegenteil: Die Differenzblindheit des Universalismus läuft das Risiko, privilegierte Subjekte in ihrem ungestörten Selbstbezug zu belassen und damit politische Erfahrungen noch unwahrscheinlicher zu machen als sie ohnehin schon sind (Medina 2013, S. 149-154). So kommt Rancière zum gleichen Selbstwiderspruch wie französische Republikaner*innen, die die ,identitären‘ Diskurse nicht-weißer Akteur*innen kritisieren, aber kein Problem damit haben, ihren Universalismus als französische Eigenheit zu verteidigen. ${ }^{15}$ An diesem Punkt zeigt sich in aller Deutlichkeit, dass Universalismus nicht immer und überall eine Desidentifizierung von polizeilicher Identität erreicht; er kann sogar selbst zum Polizeimechanismus werden. Anstatt also Desidentifizierung und Universalität gleichzusetzen, müsste zwischen kontextspezifischen Subjektivierungsformen unterschieden werden. So kann ein als ,weiß' rassifiziertes Subjekt kaum durch einen abstrakten Universalismus ,verlernen, ,weiß“ zu sein“, sondern, wie Fanon (2008, S. 111-114) betont, dialektisch, in der Begegnung mit der Selbstaffirmation des Nicht-Weißen (Vergès 2005, S. 63).

Auf eine differenziertere Sicht zielt Medinas (2013, S. 27-30) Idee der „epistemischen Reibung“ (epistemic friction), die insofern der Rancièreschen Desidentifizierung ähnelt, als dass sie das Infragestellen von Selbstidentität als Dimension von Demokratie betont, im Gegensatz zu Rancière aber Desidentifizierung als sozial-historisch situierten Prozess zwischen unterschiedlich positionierten epistemischen Subjekten versteht. Bei Rancière könnte es vor allem seine ästhetische Theorie sein, die die Möglichkeit eröffnet, Medina in eine empirisch anschlussfähige Analyse zu folgen. So hat Rancière (2013a, S. 16-20) zwischen „Kunstregimen“ (,régimes de

15 Eine weitaus kritischere Betrachtung der Wechselwirkungen universalistischer Diskurse und rassifizierter Machtstrukturen findet sich hingegen bei Balibar 2017. 
l'art”) unterschieden: dem ethischen, dem repräsentativen und dem ästhetischen. Dabei entspricht insbesondere der Übergang vom repräsentativen zum ästhetischen Regime nicht nur einer Unterscheidung zwischen referentieller und abstrakter Kunst, sondern auch einem historischen Prozess und dem, was im Anschluss an MerleauPonty (2015, S. 161-165) eine „Institution“16 genannt werden kann: einem „Regime des Wahrnehmungsvermögens [perception], der Sinneswahrnehmung [sensation] und der Interpretation von Kunst“ (Rancière 2013a, S. x) sowie einem „Regime der Historizität" (Rancière 2013b, S. 13). Damit ermöglicht die Kategorie des régime esthétique auch grundsätzlich eine Unterscheidung zwischen historisch situierten Polizeiregimen. Einer solchen Interpretation folgend, die Rancière in der Tradition von Merleau-Ponty, Lefort und Castoriadis verortet, würde es wiederum möglich, die Postkolonie als régime zu begreifen - als eine instituierte Konfiguration ästhetischer Erfahrung, deren Spezifik u.a. in der polizeilichen Funktionsweise universalistischer Diskurse besteht.

\section{Fazit: Universalismus als Polizei, Solidarität als Politik}

Trotz Rancières eigener Behauptung, sich niemals mit Fragen „postkolonialer Identitäten" befasst zu haben (Dasgupta 2008, S. 76), erscheint La cause de l'autre als ein wichtiger Beitrag zum Nachdenken über Solidarität unter den Bedingungen der Postkolonie. Gegen die Vorstellung anti-kolonialer Politik als Kampf um die Selbstidentität eines unterdrückten Anderen entwickelt Rancière einen dezidiert politischen Begriff der Solidarität als Prozess der Selbstverfremdung unter Gleichen. Aus Rancières Sicht eröffnet die Desidentifizierung von der eigenen, vermeintlich stabilen Selbstidentität die Möglichkeit einer citoyenneté politique jenseits ,polizeilich zugewiesener Identitäten. Solche politische Bürger*innenschaft bringt bestenfalls einen Universalismus der Zwischenräume hervor, für den eine universelle Gleichheit nicht in transzendentalen Höhen angesiedelt ist, sondern in der Realität des politischen Handelns. Doch so sehr egalitäre Politik die polizeiliche Hierarchie unterbrechen mag, so bleibt sie stets „unrein“ und mit den ihr entgegengesetzten Herrschaftsstrukturen „verknotet“ (Chambers 2013; Woodford 2016). Wie Rancière in seiner Antwort auf Honneth darlegt, führt ,die Beachtung von Verwerfungen und Asymmetrien“ nicht zwangsläufig zu „Relativismus“, sondern sie kann auch die Grundlage bilden für eine

„anspruchsvollere Form des Universalismus: einer Form des Universalismus, die sich nicht auf bloße Spielregeln beschränkt, sondern einen permanenten Kampf bezeichnet

16 Balibar (2016, S. 142) suggeriert, dass Merleau-Ponty eine zentrale, kaum thematisierte Inspiration für Rancières Politikverständnis bilde. Zu Rancière und Merleau-Ponty siehe auch Plot 2014, S. 95-101. 
[...], eine Erfindung von Verfahren, die dafür sorgen, dass ein existierendes Universelles seine eigenen Grenzen konfrontiert und überwindet" (Deranty/Genel 2016, S. 84).

Doch genau diese Problematisierung der Grenzen universalistischer Diskurse im symbolischen Raum der französischen Postkolonie ist bei Rancière nicht zu finden. Sein Universalismus bleibt unsensibel für die Verstrickungen mit einer Republikanismustradition, die sich zwar als identitätsübergreifend ausgibt, jedoch gerade in dieser Abstraktion anti-politisch operiert: Unter postkolonialen Bedingungen schlägt Rancières Politikbegriff um in einen Universalismus als Polizei, der privilegierte Subjekte in ihrem reibungslosen Selbstsein bestätigt und damit ein auf gegenseitige Selbstverfremdung angewiesenes politisches Handeln zusätzlich erschwert. Dadurch verspielt Rancière seine wertvollen Einsichten in die notwendige Beziehung zwischen Alterität und politischer Solidarität. Die Methode des emanzipatorischen Apriorismus, der die universelle Gleichheit als unbegründbare These einführt, erweist sich als Symptom einer Meta-Blindheit für die konkreten Bedingungen und Effekte universalistischer Diskurse. Schärfer formuliert: Rancièresche Politik wird selbst zur Polizei, insofern sie ihre Differenzblindheit in einer spezifisch französischen Universalismus-Tradition begründet, postkoloniale und rassifizierte Machtstrukturen systematisch ausblendet und gerade dadurch perpetuiert. Trotz seines intensiven Nachdenkens über das französisch-algerische Verhältnis, verweigert sich Rancière einer ,,intimeren Kolonialgeschichte“ (Stoler 2011, S. 131), erklärt postkoloniale Studien in Frankreich für inexistent und vermeidet die kritische Auseinandersetzung mit der kolonialen Dimension historisch instituierter Universalismen im Plural, wie sie Balibar in den letzten Jahrzehnten entwickelt hat. Mit dieser Kritik ist Rancières theoretische Provokation aber noch nicht von der Hand gewiesen, sondern verlangt nach einem gehaltvolleren Institutionsbegriff und einem Nachdenken über die Möglichkeit nicht-polizeilicher Formen empirischer Sozialforschung.

\section{Literatur}

Abbas, Nabila, 2019: Jacques Rancière. In: Comtesse, Dagmar/Flügel-Martinsen, Oliver/ Martinsen, Franziska/Nonhoff, Martin (Hg.): Radikale Demokratietheorie, Frankfurt a.M., S. 388-400.

Balibar, Étienne, 1992: Le mot race n'est pas „de trop“ dans la Constitution française. In: Mots. Les langages du politique 33, S. 241-256.

Balibar, Étienne, 1997: Algérie, France: une ou deux nations?. In: Lignes 1, 30, S. 5-22.

Balibar, Étienne, 2016: Des Universels. Essais et conférences. Paris.

Balibar, Étienne, 2017: L'universalisme ne rassemble pas, il divise. In: Le Monde, 9.2.2017. Unter: https://www.lemonde.fr/idees/article/2017/02/09/etienne-balibar-1-universel-ne-rass emble-pas-il-divise_5077178_3232.html, download am 18.10.2019. 
Beltrán, Cristina, 2009: Going Public. Hannah Arendt, Immigrant Action, and the Space of Appearance. In: Political Theory 37, 5, S. 595-622.

Bentouhami, Hourya, 2017: Notes pour un féminisme marron. Du corps-doublure au corps propre. In: Comment s'en sortir? 5, S. 108-125.

Bosteels, Bruno, 2010: Archipolitics, Parapolitics, Metapolitics. In: Deranty, Jean-Philippe (Hg.): Jacques Rancière. Key Concepts. New York, S. 80-92.

Brunet, Jean-Paul, 1999: Police contre FLN. Le Drame d'octobre 1961. Paris.

Butler, Judith, 2000: Restaging the Universal. Hegemony and the Limits of Formalism. In: Butler, Judith/Laclau, Ernesto/Žižek, Slavoj: Contingency, Hegemony, Universality. Contemporary Dialogues on the Left, London/New York, S. 11-43.

Castoriadis, Cornelius, 1999: L'institution imaginaire de la société. Paris.

Chakrabarty, Dipesh, 2009: Provincializing Europe. Postcolonial Thought and Historical Difference. Princeton.

Chambers, Samuel/O'Rourke, Michael, 2009: Jacques Rancière on the Shores of Queer Theory. In: borderlands 8, 2, S. 1-19.

Chambers, Samuel, 2013: The Lessons of Rancière. Oxford.

Conklin, Alice, 1997: A Mission to Civilize. The Republican Idea of Empire in France and West Africa. 1895-1930. Stanford.

Deranty, Jean-Philippe, 2014: Regimes of the arts. In: Deranty, Jean-Philippe (Hg.): Jacques Rancière: Key Concepts. London, S. 116-133.

Deranty, Jean-Philippe/Genel, Katia (Hg.), 2016: Recognition or Disagreement? A Critical Encounter on the Politics of Freedom, Equality, and Identity. New York.

Dorlin, Elsa, 2017: Se Défendre. Une philosophie de la violence. Paris.

Fanon, Frantz, 2008: Black Skin, White Masks. New York.

Fjeld, Anders, 2018: Jacques Rancière. Pratiquer l'égalité. Paris.

Flügel-Martinsen, Oliver, 2015: Die Normativitätsbegründungsfalle. Die unterschätzte Bedeutung befragender und negativer Kritikformen in der Politischen Theorie und der Internationalen Politischen Theorie. In: Zeitschrift für Politische Theorie 6, 2, S. 189-206.

Gündoğdu, Ayten, 2017: Disagreeing with Rancière. Speech, Violence, and the Ambiguous Subjects of Politics. In: Polity 49, 2, S. 188-219.

Harbi, Mohammed/Stora, Benjamin (Hg.), 2004: La guerre d'Algérie: 1954-2004. La fin de l'amnésie. Paris.

Havercroft, Jonathan/Owen, David, 2016: Soul-Blindness, Police Orders and Black Lives Matter. Wittgenstein, Cavell, and Rancière. In: Political Theory 44, 6, S. 739-763.

Lefort, Claude, 1986: Essais sur le politique. Paris.

Marchart, Oliver, 2000: Division and Democracy. On Claude Lefort's Post-foundational Political Philosophy. In: Filozofski vestnik XXI, 2, S. 51-82.

Marchart, Oliver, 2010: Die politische Differenz. Frankfurt a.M.

May, Todd, 2009: There are no Queers. Jacques Rancière and post-identity politics. In: borderlands 8, 2, S. 1-17.

May, Todd, 2010: Contemporary Political Movements and the Thought of Jacques Rancière. Equality in Action. Edinburgh.

Mbembe, Achille, 2001: On the Postcolony. Berkeley/Los Angeles. 
McNay, Lois, 2014: The Misguided Search for the Political. London.

Medina, José, 2013: The Epistemology of Resistance. Gender and Racial Oppression, Epistemic Injustice, and Resistant Imaginations. Oxford.

Merleau-Ponty, Maurice, 201): L'institution, la passivité. Notes de cours au Collège de France (1954-1955). Mit einem Vorwort von Claude Lefort. Paris.

Mouffe, Chantal, 2005: On the Political. London/New York.

Myers, Ella, 2016: Presupposing equality: The trouble with Rancière's axiomatic approach. In: Philosophy \& Social Criticism 42, 1, S. 45-69.

Péju, Marcel/Péju, Paulette/Manceron, Gilles, 2011: Le 17 octobre des Algériens, suivi de. La triple occultation d'un massacre. Paris.

Peyroulou, Jean-Pierre, 2008: Le cas de Sétif-Kherrata-Guelma (Mai 1945). In: Violence de masse et Résistance. Réseau de recherche. 21.3.2008. Unter: http://bo-k2s.sciences-po.fr/m ass-violence-war-massacre-resistance/fr/document/le-cas-de-sa-tif-kherrata-guelma-mai-19 45, download am 15. 2. 2020.

Plot, Martín, 2014: The Aesthetico-Political. The Question of Democracy in Merleau-Ponty, Arendt, and Rancière. London/New York.

Rancière, Jacques, 1997: La cause de l'autre. In: Lignes 1, 30, S. 36-49.

Rancière, Jacques, 1998: Aux bords du politique. Paris.

Rancière, Jacques, 2000: Des régimes de l'art et du faible intérêt de la notion de modernité. In: Rancière, Jacques: Le partage du sensible. Esthétique et politique. Paris.

Rancière, Jacques, 2006: Hatred of Democracy. London/New York.

Rancière, Jacques, 2009: A few remarks on the method of Jacques Rancière. In: Parallax 15, 3, S. 114-123.

Rancière, Jacques, 2013a: The Politics of Aesthetics. London/New York.

Rancière, Jacques, 2013b: Aisthesis. Scenes from the Aesthetic Regime of Art. London/New York.

Rancière, Jacques, 2014a: Das Unvernehmen. Politik und Philosophie. Frankfurt a.M.

Rancière, Jacques, 2014b: Moments Politiques. Interventions 1977-2009. New York.

Rancière, Jacques, 2016: Interview Thomas Claviez/Dietmar Wetzel mit Jacques Rancière. In: Claviez, Thomas/Wetzel, Dietmar: Zur Aktualität von Jacques Rancière: Einleitung in sein Werk, Berlin, S. 153-171.

Rancière, Jacques, 2019: Les vertus de l'inexplicable. À propos des ,gilets jaunes.' In: AOC, 8.1.2019. Unter: https://aoc.media/opinion/2019/01/08/vertus-de-linexplicable-a-propos-gil ets-jaunes/, download am 18. 10. 2019.

Russell, Matheson/Montin, Andrew, 2015: The Rationality of Political Disagreement. Rancière's Critique of Habermas. In: Constellations 22, 4, S. 543-554.

Seelow, Soren, 2011: 17 octobre 1961. In: Le Monde, 17.10.2010. Unter: https://www.lemond e.fr/societe/article/2011/10/17/17-octobre-1961-ce-massacre-a-ete-occulte-de-la-memoirecollective_1586418_3224.html, download am 18.10.2019. 
Schaap, Andrew, 2011: Enacting the Right to Have Rights. Jacques Rancière's Critique of Hannah Arendt. In: European Journal of Political Theory 10, 1, S. 22-45.

Spivak, Gayatri Chakravorty, 2006: Subaltern Studies. Deconstructing Historiography. In: Spivak, Gayatri Chakravorty: In Other Worlds. Essays in Cultural Politics. New York/London, S. 179-196.

Stoler, Ann Laura, 2011: Colonial Aphasia. Race and Disabled Histories in France. In: Public Culture 23, 1, S. 121-156.

Toscano, Alberto, 2018: The Name of Algeria: French Philosophy and the Subject of Decolonization. In: Viewpoint Magazine. Unter: https://www.viewpointmag.com/2018/02/01/nam e-algeria-french-philosophy-subject-decolonization/, download am 18.10.2019.

Theoharis, Jeanne, 2013: The Rebellious Life of Mrs. Rosa Parks. Boston.

Vergès, Françoise, 2005: „Le Nègre n'est pas. Pas plus que le Blanc.“ Frantz Fanon, esclavage, race, et racisme. In: Actuel Marx 2, 38, S. 45-63.

Vergès, Françoise, 2019: Un féminisme décolonial. Paris.

Wahnich, Sophie, 2016: Nuit Debout. In: L'Homme \& La Société 20, 200, S. 7-12.

Wall, Ilan Rua, 2012: Tunisia and the Critical Legal Theory of Dissensus. In: Law and Critique 23, 3, S. 219-236.

Wilder, Gary, 2015: Freedom Time. Negritude, Decolonization, and the Future of the World. Durham.

Woodford, Claire, 2016: Disorienting Democracy. Politics of emancipation. Abingdon/New York.

Zerilli, Linda, 2005: Feminism and the Abyss of Freedom. Chicago. 